\title{
Proteins in plasma as a potential biomarkers diagnostic for pelvic organ prolapse
}

\author{
Tao Wang ${ }^{1}$, Yuqing Liu ${ }^{1}$, Ling Mei ${ }^{2}$, Tao Cui ${ }^{2}$, Dongmei Wei ${ }^{2}$, Yueyue Chen ${ }^{2}$, Xiaoli Zhang ${ }^{1}$, Linbo Gao ${ }^{1}$, \\ Shihong Zhang ${ }^{3}$, Lanfang Guo ${ }^{3}$, Pei Yang ${ }^{3}$, Xiaoyu Niu ${ }^{2}$
}

${ }^{1}$ Center for Translational Medicine, Key Laboratory of Birth Defects and Related Diseases of Women and Children (Sichuan University), Ministry of Education, Department of Obstetrics and Gynecology, West China Second University Hospital, Sichuan University, Chengdu, China; ${ }^{2}$ Department of Obstetrics and Gynecology, Key Laboratory of Birth Defects and Related Diseases of Women and Children (Sichuan University), Ministry of Education, West China Second University Hospital, Sichuan University, Chengdu, China; ${ }^{3}$ Health Management Center, West China Second University Hospital, Sichuan University, Chengdu, China

Contributions: (I) Conception and design: T Wang, X Niu; (II) Administrative support: T Wang, X Niu; (III) Provision of study materials or patients: Y Liu, L Mei, T Cui, D Wei, Y Chen, X Zhang, L Gao, S Zhang, L Guo, P Yang; (IV) Collection and assembly of data: T Wang, Y Liu, X Zhang, L Gao; (V) Data analysis and interpretation: T Wang, Y Liu, L Gao, X Niu; (VI) Manuscript writing: All authors; (VII) Final approval of manuscript: All authors.

Correspondence to: Dr. Xiaoyu Niu, MD. Department of Obstetrics and Gynecology, West China Second University Hospital, Sichuan University, 20 Ren Min Nan Lu, Chengdu 610041, China. Email: niuxy@scu.edu.cn.

\begin{abstract}
Background: Pelvic organ prolapse (POP) is the most common and widespread type of female pelvic floor dysfunction disease (PFD). At present, the diagnosis of POP is mainly based on a complicated systematic evaluation of the clinical phenotype, medical history, and relevant functional examinations. Rapid and simple tests that are based on biochemical biomarkers that surpass the sensitivity and specificity of the current methods for the diagnosis of POP will greatly facilitate the timely diagnosis and treatment of women with POP.
\end{abstract}

Methods: A protein array was used to screen plasma samples collected from healthy controls and women with POP. Enzyme-linked immunosorbent assays (ELISAs) were used to determine the levels of three novel and potentially useful analytes: heat shock protein 10 (HSP10), zinc finger CCCH domain-containing protein 8 ( $\mathrm{ZC} 3 \mathrm{H} 8)$, and unc-45 myosin chaperone A (UNC45A). We then determined the diagnostic value of each of these analytes as potential diagnostic biomarkers for clinical application.

Results: The mean levels of HSP10, ZC3H8, and UNC45A, were lower in the plasma samples from 76 patients with POP than in 56 samples from healthy controls $(\mathrm{P}<0.05)$. Comparisons between patients with POP and healthy controls demonstrated the sensitivity and specificity of HSP10 (73.7\% and 71.4\%), ZC3H8 (71.1\% and $62.5 \%)$, and UNC45A (70.7\% and 62.5\%).

Conclusions: Analysis indicated that plasma levels of HSP10, ZC3H8, and UNC45A, are sensitive and specific biomarkers for the diagnosis of POP.

Keywords: Biomarker; pelvic organ prolapse (POP); heat shock protein 10 (HSP10); antibody array; diagnostic value

Submitted Apr 01, 2021. Accepted for publication Jun 12, 2021.

doi: $10.21037 /$ atm-21-1607

View this article at: https://dx.doi.org/10.21037/atm-21-1607

\section{Introduction}

Pelvic organ prolapse (POP) is the most common and widespread female pelvic floor dysfunction disease (PFD) and is often accompanied by structural and functional defects of the pelvic supporting tissues. POP can have significant effects on a woman's quality of life, along with their physical and mental health (1). The global incidence of POP is approximately $20 \%$ and is increasing on an annual 
basis $(2,3)$. Multiple factors including age, menopause, parity, obesity (4), hysterectomy, and connective tissue disorders have been reported to be in association with POP development $(5,6)$. Age more than 55 years old and natural childbirth history were reported as two independent risk factors for POP, suggesting the existence of long-term pathology. However, the underlying molecular mechanisms of POP are still unclear.

At present, the diagnosis of POP is mainly based on clinical phenotype, medical history, and relevant functional examinations. However, there is a clear absence of specific biomarkers for functional molecules in the early stages that can reflect the long-term pathological progression. Therefore, it is very challenging to identify and evaluate patients with POP, and perform appropriate interventions in a timely manner. Therefore, a rapid and simple test that is based on highly accurate biomarkers that surpass the sensitivity and specificity of the current methods used for the diagnosis of POP will significantly enhance our ability to treat women who are suspected of having POP.

Deng et al. utilized ultra-high performance liquid chromatography-quadrupole time-of-flight mass spectrometry (UHPLC/Q-TOF-MS) to comprehensively investigate serum and urine 17 metabolomes of POP patients and controls and found that six metabolites including glycerophosphocholine (GPC), 1-methyladenosine, maleic acid, L-pyroglutamic acid, inosine, and citrate are significantly changed in both serum and urine samples from patients with POP (7). Zou et al. reported that the higher levels of matrix metalloproteinase (MMP)-2 and MMP-9 and lower levels of tissue inhibitor of metalloproteinase (TIMP)-2 in the plasma of Caucasian women may indicate a greater tendency for collagenolysis and weaker connective tissue with increased risk of developing PFDs, and they may potentially serve as biomarkers for pelvic floor tissue integrity (8).

In this study, we used an antibody array as a screening tool and identified three proteins heat shock protein 10 (HSP10), zinc finger CCCH domain-containing protein 8 (ZC3H8), and unc-45 myosin chaperone A (UNC45A), that exhibited significantly different levels when compared between patients with POP and healthy controls. Based on these findings, we demonstrated that these proteins represent good diagnostic biomarkers for POP.

We present the following article in accordance with the STARD reporting checklist (available at https://dx.doi. org/10.21037/atm-21-1607).

\section{Methods}

\section{Subjects}

The study was conducted in accordance with the Declaration of Helsinki (as revised in 2013). The study was approved by the Research Medical Ethics Committee of West China Second University Hospital, Sichuan University (2020121) and informed consent was taken from all individual participants. In total, 132 women were enrolled in this study between September 2019 and September 2020. Women with malignant tumors, fibromyoma, pelvic masses, and mental abnormalities were excluded. Of the women included, 76 were diagnosed with POP and 56 women without POP were recruited as healthy controls (Table 1). As presented in Table 1, age of healthy controls was between 45 and 75 years, with a mean age of 57 years. Age of patients with POP was between 41 and 83 years, with a mean age of 63 years. Thirty-two women with POP were parity with once and 44 women with POP were parities with equal and more than two times, while 22 non-POP women were parity with once and 33 non-POP women were parities with equal and more than two times.

The clinical description of pelvic floor anatomy was determined during physical examinations of the external genitalia and vaginal canal. POP was confirmed according to the following criteria: (I) symptoms attributed to POP, such as a bothersome bulge and pressure symptoms, sexual dysfunction, lower urinary tract dysfunction, or defecatory dysfunction; and (II) a POP quantification (POP-Q) examination was applied to judge the grade of POP (9). POP grade II was defined as when the leading edge of POP was approximately $1 \mathrm{~cm}$ inside the hymen margin to $1 \mathrm{~cm}$ outside the hymen. POP grade III was defined as when the leading edge of POP was beyond the level of $1 \mathrm{~cm}$ above the hymen (10). POP grade IV was defined as when the farthest end of POP exceeded the hymen margin and was greater than the total length of the vagina minus $2 \mathrm{~cm}$. Non-POP healthy controls were recruited according to the following criteria: (I) women accepted physical examination in our hospital and showed normal results with regards to medical reports, biochemical tests, malignant tumors, pelvic masses, mental abnormalities, and PFD; (II) there were no complaints of symptoms such as sexual dysfunction, lower urinary tract dysfunction, or defecatory dysfunction; and (III) women were diagnosed as non-POP by the POP-Q examination. 
Table 1 Clinical characteristics patients with POP and healthy controls

\begin{tabular}{lcc}
\hline Parameter & Healthy controls $(n=56)$ & Patients with POP $(n=76)$ \\
\hline Age (year) & & $62.74 \pm 9.33^{*}$ \\
Mean \pm SD & $57.00 \pm 8.67$ & $41-83$ \\
Range & $45-75$ & \\
Parity & & $32(42.1)$ \\
1 & $22(39.3)$ & $44(57.9)$ \\
$\geq 2$ & $33(60.7)$ & $30^{\star}$ \\
Hypertension & 10 & 11 \\
Diabetes & 4 & $7(9.2)^{\star}$ \\
POP grade & 0 & $59(77.6)^{\star}$ \\
II & 0 & $10(13.2)^{\star}$ \\
III & 0 & \\
IV & 0 &
\end{tabular}

Data are shown as $\mathrm{n}(\%)$ or mean $\pm \mathrm{SD} .{ }^{*}$, statistically significant $(\mathrm{P}<0.05$, women with POP vs. healthy controls). POP, pelvic organ prolapse; SD, standard deviation.

\section{Sample collection}

Blood samples were collected from 76 patients with POP and 56 healthy controls. From each woman, we acquired $1 \mathrm{~mL}$ of venous blood which was placed into a sterile ethylene diamine tetraacetic acid (EDTA)-containing vacutainer tube. Samples were then centrifuged at 1,600 rpm at $4{ }^{\circ} \mathrm{C}$ for $10 \mathrm{~min}$. The resultant plasma was then collected, aliquoted, and stored at $-80^{\circ} \mathrm{C}$ to await subsequent analysis.

\section{Screening assay using antibody array}

Three plasma samples from 76 patients with POP and three samples from 56 healthy controls were screened using the L-Series human antibody array 2000 membrane kit, which is a combination of human L507, L-493, L-3 and L-4 arrays (AAH-BLM-2000B, RayBiotec Inc., Norcross, GA, USA). This array could simultaneously detect 2,000 soluble human proteins. Experiments were conducted in accordance with the manufacturer's guidelines. In brief, three plasma samples from each group were mixed in equal volumes. Each mixture was then dialyzed in dialysis buffer on a stirring plate for at least $3 \mathrm{~h}$ at $4{ }^{\circ} \mathrm{C}$. The dialyzed plasma samples were then labeled with biotin at room temperature (RT) for $30 \mathrm{~min}$. The array membranes were then incubated in blocking buffer at RT for $1 \mathrm{~h}$. After removing the blocking buffer, the biotin-labeled samples were diluted with blocking buffer, placed onto the membrane array and incubated at RT for $4 \mathrm{~h}$. Following incubation, the sample fluid was discarded, and the membranes were washed three times. HRP-conjugated streptavidin was then added and incubated at RT for $2 \mathrm{~h}$. Membranes were then washed three times in $2 \mathrm{~mL}$ of washing buffer. Signals were then detected and visualized by the addition of a detection mixture and the application of a chemiluminescence imaging system (ChemiDoc ${ }^{\mathrm{TM}}$ MP Imaging System, BioRad Laboratories, Hercules, CA, USA).

\section{Enzyme-linked immunosorbent assays (ELISAs)}

The levels of soluble HSP10, ZC3H8, utrophin (UTRN), UNC45 A, and unc-5 homolog 4 (UNC5H4), were determined in duplicate plasma samples by commercial ELISA kits purchased from Abbexa Ltd. (Cambridge, UK) and Raybiotech, Inc. (Norcross, GA, USA). Experiments were conducted in accordance with the manufacturer's guidelines. In brief, diluted plasma samples were added into duplicate wells and mixed with $50 \mu \mathrm{L}$ of HRP-conjugate per well in a 96-well plate. After incubation at RT for $2 \mathrm{~h}$ on a microplate shaker rotating at $100 \mathrm{rpm}$, the plate was then washed. We then added $100 \mu \mathrm{L}$ of tetramethylbenzidine (TMB) Substrate Solution to each well and incubated the plates at RT for approximately $10 \mathrm{~min}$; this process initiated the development of color. Reactions were then terminated and the optical absorbance of each well was read immediately on a spectrophotometer (Infinite M200, Tecan Trading AG, Switzerland) using $450 \mathrm{~nm}$ as the primary wavelength. The levels of each analyte were then determined by referring to a standard curve generated simultaneously on the same plate. Samples showing levels higher than the detection limits of the kits were diluted and re-measured. In our laboratory, the intra-assay and inter-assay coefficients of variation were $<10 \%$ for all three assays.

\section{Statistical analysis}

The plasma levels of HSP10, ZC3H8, UTRN, UNC45A, and $\mathrm{UNC} 5 \mathrm{H} 4$, were expressed as means \pm standard deviation (SD). The statistical significance of the findings was evaluated by the Student's $t$-test or by one-way analysis of variance (ANOVA) in Prism 8 software (GraphPad Software, San Diego, CA, USA). A P value $<0.05$ was 
Table 2 Plasma levels of HSP10, ZC3H8, UTRN, UNC45A, and UNC5H4, in patients with POP and healthy controls

\begin{tabular}{lccccc}
\hline \multirow{2}{*}{ Group } & \multicolumn{4}{c}{ Plasma levels $(\mathrm{ng} / \mathrm{mL})$} \\
\cline { 2 - 6 } & HSP10 & ZC3H8 & UTRN & UNC45A & UNC5H4 \\
\hline Healthy controls $(\mathrm{n}=12)$ & $5.43 \pm 2.86$ & $22.70 \pm 15.78$ & $37.78 \pm 23.70$ & $2.40 \pm 1.17$ & $0.00 \pm 0.00$ \\
Patients with POP $(\mathrm{n}=24)$ & $3.21 \pm 2.63^{*}$ & $14.07 \pm 17.29^{*}$ & $28.30 \pm 28.26$ & $1.40 \pm 1.71^{\star}$ & $1.53 \pm 5.47^{*}$ \\
\hline
\end{tabular}

Data are shown as mean $\pm \mathrm{SD}$. *, statistically significant $(\mathrm{P}<0.05$, compared with the plasma of patients with POP). HSP10, heat shock protein 10; ZC3H8, zinc finger $\mathrm{CCCH}$ domain-containing protein 8; UTRN, utrophin; UNC45A, unc-45 myosin chaperone A; UNC5H4, unc5 homolog 4; POP, pelvic organ prolapse; SD, standard deviation.

considered to be statistically significant. The plasma levels of HSP10, ZC3H8, and UNC45A, were used to create a threshold that indicated the maximal sensitivity and specificity for the diagnosis of POP, as determined by receiver operator characteristic (ROC) curves.

\section{Results}

\section{Screening of plasma samples}

The L-Series 2000 antibody array used in this study could simultaneously detect 2,000 cytokines and related proteins in a given sample. The results were semi-quantitative when assessed by the density of each analyte spot. Multiple analytes were detected positively in the mixed sample created from each group. Among the positive analytes, the plasma levels of HSP10, ZC3H8, UTRN, UNC45A, and $\mathrm{UNC} 5 \mathrm{H} 4$, were significantly decreased in the samples from patients with POP. The essential feature of diagnostic biomarkers is the fact that they exhibit significant differences when compared between samples from patients with disease and those from healthy controls.

\section{Plasma levels of HSP10, ZC3H8, and UNC45A, in samples from patients with POP and healthy controls}

Following ELISA screening, we focused only on UNC45A, UNC5H4, HSP10, ZC3H8, and UTRN because these proteins appeared to be more promising than the other analytes. As presented in Table 2, ELISA assays detected quantitative differences in the levels of HSP10, ZC3H8, UTRN, UNC45A, and UNC5H4, between 24 patients with POP and 12 healthy controls. The mean levels of HSP10, ZC3H8, and UNC45A, in samples from patients with POP were $3.21 \pm 2.63,14.07 \pm 17.29$, and $1.40 \pm 1.71$ $\mathrm{ng} / \mathrm{mL}$; this compared to $5.43 \pm 2.86,22.70 \pm 15.78$, and $2.40 \pm 1.17 \mathrm{ng} / \mathrm{mL}$, in the healthy controls. The plasma levels of UTRN were not significantly different when compared between the two types of samples. We observed higher plasma levels of UNC5H4 in samples from patients with POP, although some samples in each group did not show a detectable level of this protein. Plasma levels of HSP10, $\mathrm{ZC} 3 \mathrm{H} 8$, and UNC45A, were significantly lower in the samples from patients with POP than in the samples from healthy controls. Consequently, our subsequent analysis focused on only HSP10, ZC3H8, and UNC45A.

Next, we evaluated the diagnostic value of these three biomarkers by recruiting 76 POP patients and 56 healthy controls. As depicted in Figure 1, the levels of HSP10, ZC3H8, and UNC45A, in the plasma samples of POP patients were $2.07 \pm 3.49,13.94 \pm 28.42$, and $4.73 \pm 21.40 \mathrm{ng} / \mathrm{mL}$, respectively. These were significantly lower than the plasma levels in healthy controls with $4.16 \pm 3.85,23.40 \pm 22.43$, and $6.04 \pm 13.02 \mathrm{ng} / \mathrm{mL}$, respectively $(\mathrm{P}<0.05)$.

Our data showed that aging was associated with POP. Since people older than 60 years of age are considered to be elderly, subjects in this study were grouped by age at 60 years old, as shown as Figure 2. Obviously, the plasma levels of HSP10, ZC3H8, and UNC45A, were significantly lower in the plasma samples from patients with POP compared with the control within the same age group at aged $<60$ or $>60$ years. It indicates that these biomarkers were capable to distinguish between patients or controls even they plasma concentration were related with aging. There were no significant differences in the plasma levels of these three proteins in the controls irrespective of whether the controls were aged $<60$ or $>60$ years. A similar trend was evident in the samples acquired from patients with POP.

\section{The diagnostic value of HSP10, ZC3H8, and UNC45A, for POP}

Based on the individual levels of the three target proteins in plasma samples from patients with POP and healthy 


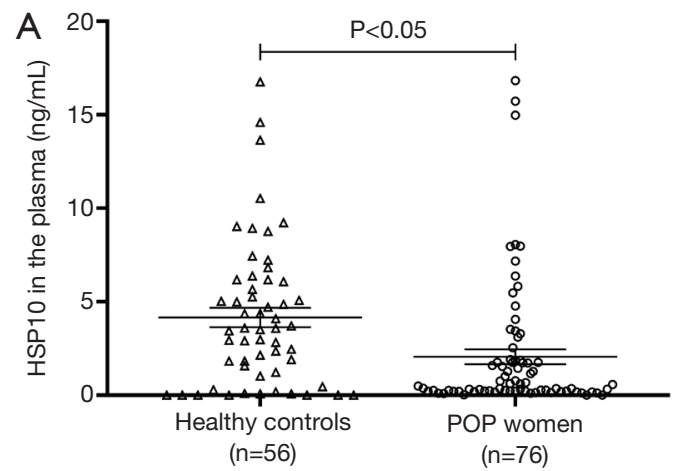

A

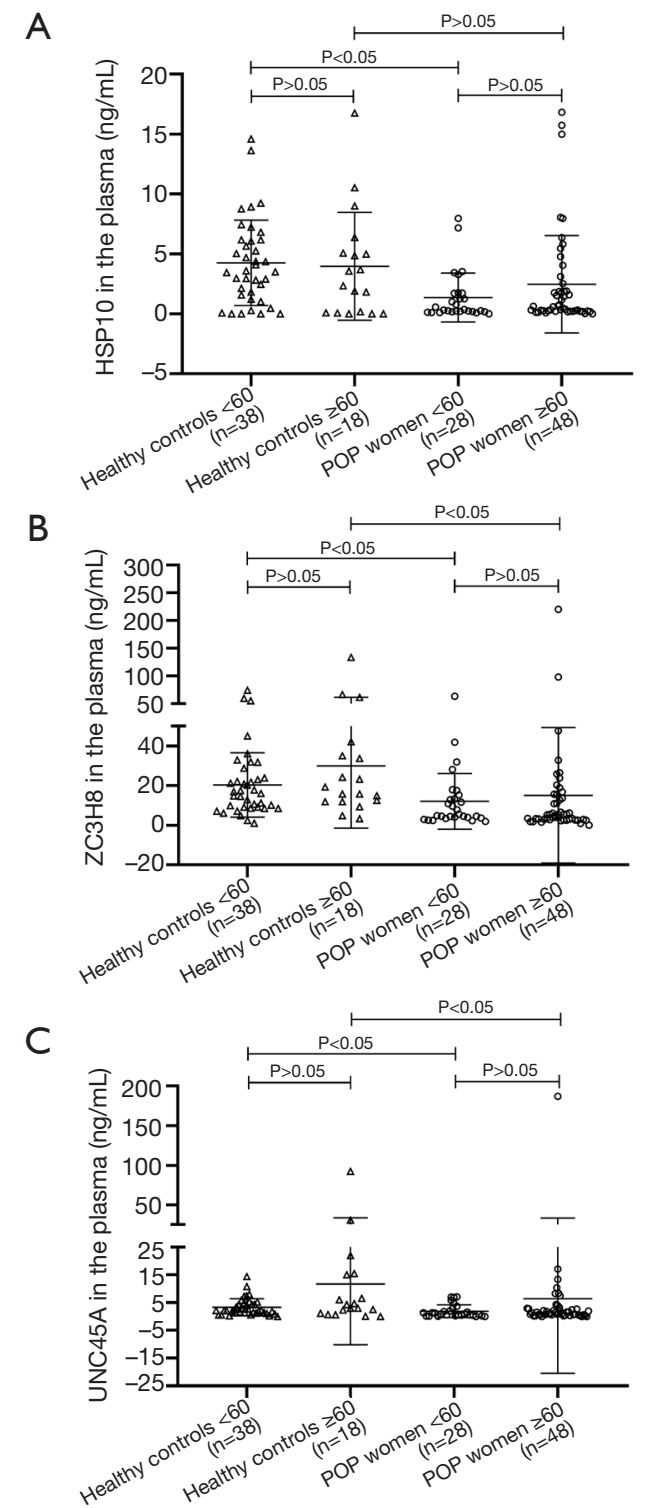

B
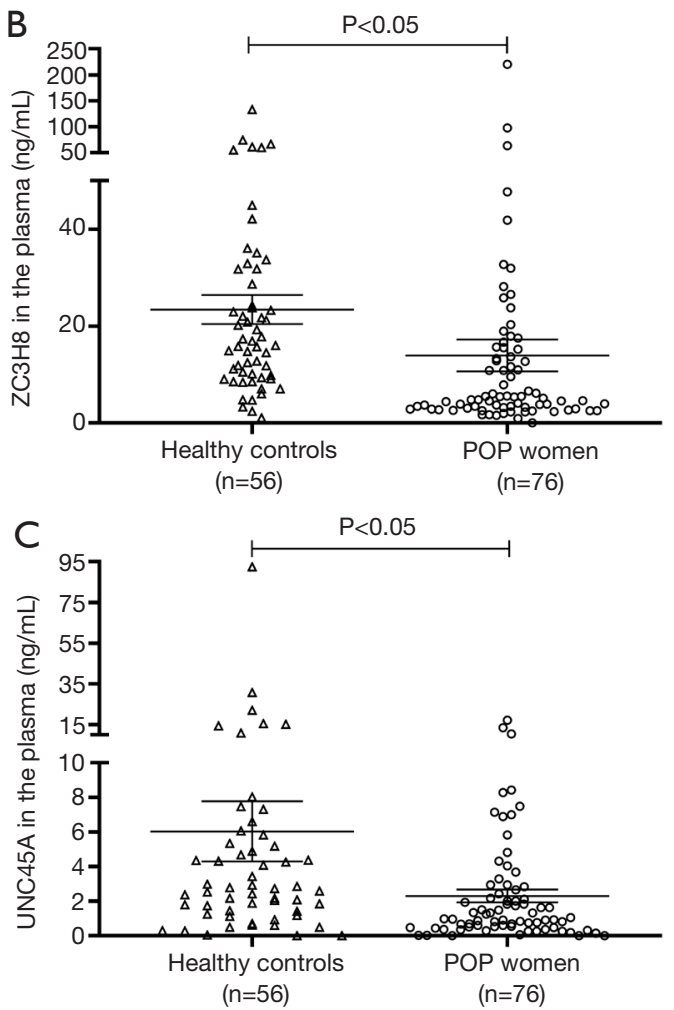

Figure 1 Differences in the plasma levels of HSP10, ZC3H8, and UNC45A, between patients with POP and healthy controls, were determined by ELISA. The three analytes were significantly reduced in POP samples when compared to control samples. (A) Differences in the plasma levels of HSP10 between patients with POP and healthy controls; (B) differences in the plasma levels of ZC3H8 between patients with POP and healthy controls; (C) differences in the plasma levels of UNC45A between patients with POP and healthy controls. Age of healthy controls was between 45 and 75 years, with a mean age of 57 years. Age of patients with POP was between 41 and 83 years, with a mean age of 63 years. $\triangle$, healthy control; $\circ$, POP woman. HSP 10 , heat shock protein 10; $\mathrm{ZC} 3 \mathrm{H} 8$, zinc finger $\mathrm{CCCH}$ domain-containing protein 8; UNC45A, unc-45 myosin chaperone A; POP, pelvic organ prolapse; ELISA, enzyme-linked immunosorbent assay.
Figure 2 Differences in the plasma levels of HSP10, ZC3H8, and UNC45A, as determined by ELISA, in patients with POP and healthy controls when grouped by age. All three analytes were significantly reduced in POP samples than in control samples within the same age group. There were no significant differences in the controls across the age groups; a similar trend was observed with the POP samples. (A) Differences in the plasma levels of HSP 10 between patients with POP and healthy controls; (B) differences in the plasma levels of $\mathrm{ZC} 3 \mathrm{H} 8$ between patients with POP and healthy controls; (C) differences in the plasma levels of UNC45A between patients with POP and healthy controls. $\triangle$, healthy control; $\circ$, POP woman. HSP10, heat shock protein 10; $\mathrm{ZC} 3 \mathrm{H} 8$, zinc finger $\mathrm{CCCH}$ domain-containing protein 8; UNC45A, unc-45 myosin chaperone A; ELISA, enzyme-linked immunosorbent assay; POP, pelvic organ prolapse. 

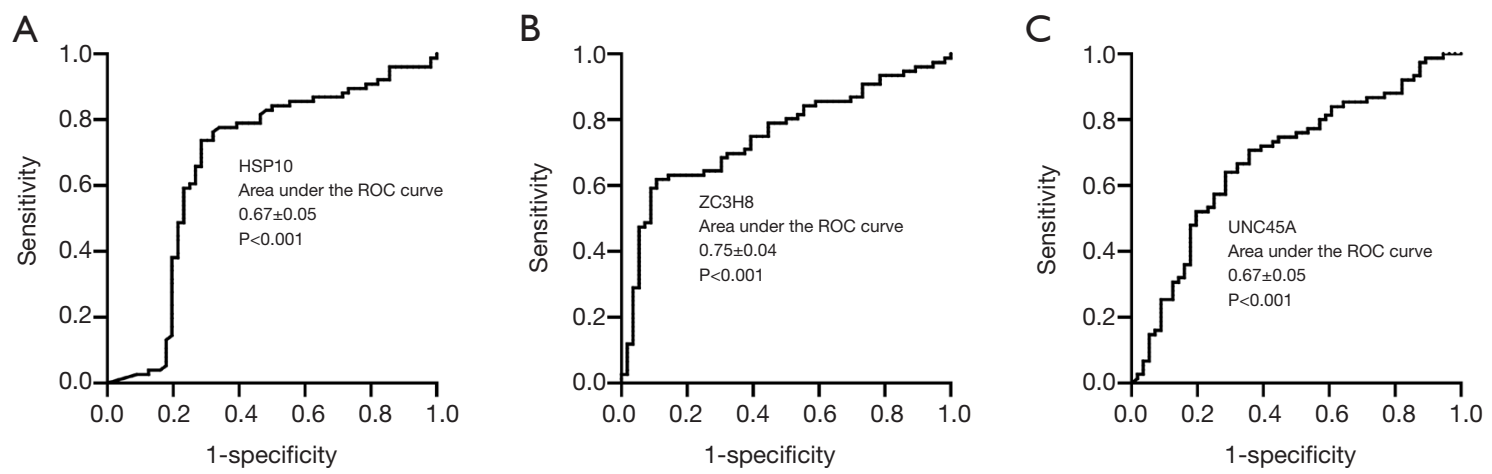

Figure 3 The diagnostic value of HSP10, ZC3H8, and UNC45A, for POP. ROC curves were plotted for each of the three analytes; all exhibited good levels of sensitivity and specificity for the diagnosis of POP. (A) The diagnostic value of HSP10 for POP; (B) the diagnostic value of ZC3H8 for POP; (C) the diagnostic value of UNC45A for POP. HSP10, heat shock protein 10; ZC3H8, zinc finger CCCH domain-containing protein 8; UNC45A, unc-45 myosin chaperone A; POP, pelvic organ prolapse; ROC, receiver operator characteristic.

Table 3 The diagnostic value of HSP10, ZC3H8, and UNC45A, for POP

\begin{tabular}{|c|c|c|c|c|c|c|}
\hline Biomarker & Cutoff (ng/mL) & \multicolumn{2}{|c|}{ Sensitivity } & \multicolumn{2}{|c|}{ Specificity } & Likelihood ratio \\
\hline HSP10 & $<1.79$ & 73.7 & 62.8 to 82.3 & 71.4 & 58.5 to 81.6 & 2.58 \\
\hline ZC3H8 & $<12.75$ & 71.1 & 60.0 to 80.0 & 62.5 & 49.4 to 74.0 & 1.90 \\
\hline UNC45A & $<2.06$ & 70.7 & 59.6 to 79.8 & 62.5 & 49.4 to 74.0 & 1.88 \\
\hline
\end{tabular}

HSP10, heat shock protein 10; ZC3H8, zinc finger CCCH domain-containing protein 8; UNC45A, unc-45 myosin chaperone A; POP, pelvic organ prolapse; $\mathrm{Cl}$, confidence interval.

controls, we next used ROC analysis to evaluate their diagnostic value. The threshold that provided maximal levels of sensitivity and specificity for the diagnosis of POP were selected as the cut off values. The results are shown in Figure 3 and Table 3. Using cut off values of 1.79, 12.75, and $2.06 \mathrm{ng} / \mathrm{mL}$, for HSP10, ZC3H8, and UNC45A, respectively, the sensitivities of all three proteins were $>70 \%$; HSP10 had the highest levels of sensitivity and specificity at $73.7 \%$ and $71.4 \%$, respectively.

\section{Discussion}

Pelvic and abdominal organs are supported by pelvic floor muscle which surrounds the opening of the urethra, vagina, and rectum. POP involves the long-term effects of pelvic floor muscle weakness, especially in postpartum women. A range of pathogenic factors, including infection, inflammation, trauma, and degenerative changes associated with aging, may play a key role in the pathogenesis of POP. Many middle-aged and elderly women suspected of having
POP often miss the opportunity for early rehabilitation and have to undergo surgery due to serious prolapse. Therefore, there is a critical need to investigate the functional; deterioration of the pelvic floor tissue accompanied by aging and the pathological factors created by aging-related diseases as these could represent important and highrisk factors for POP. Atrophy and weakness of the skeletal muscle are the main causes of POP.

HSP10 is known to be associated with the degeneration of muscle cell function. One of the main characteristics of muscle aging is a reduction in maximal tetanic force (Po). Previous research has shown that the overexpression of HSP10 prevents the age-related decline in Po and can prevent damage caused by muscle contraction, although damage that had already being incurred could not be repaired (11). Both HSP10 and HSP60 have been found in various types of muscle cells and are known to exert anti-apoptotic effects; these proteins are also related to the development of cardiomyopathy and the process of apoptosis in cardiomyocytes (12). However, prior to the 
present study there was no evidence to suggest that plasma levels of HSP10 could be used as a biochemical biomarker to facilitate the clinical diagnosis of POP.

$\mathrm{ZC} 3 \mathrm{H} 8$ has been reported with organ degeneration by inhibiting the inflammatory response in zebrafish mediated by the NF- $\mathrm{kB}$ pathway and leading the degeneration of multiple organs, including the liver, gut, and pancreas (13). Whether $\mathrm{ZC} 3 \mathrm{H} 8$ is related to the degeneration of muscle cell function in POP wasn't reported in previous research. In the present study, we found that the expression levels of $\mathrm{ZC} 3 \mathrm{H} 8$ were significantly lower in patients with POP when compared to healthy controls. The mechanisms underlying this observation need to be elucidated in further study.

UNC45A is widely expressed in various muscle cells. It is an essential protein for the assembly and function of muscle tissue. Studies have shown that UNC45A molecule is a dynamic component of actin stress fibers and acts as a myosin chaperone (14). Cells knocked out UNC45A were reported serious defects associated with fiber assembly errors that resulted in changes in cell morphology, abnormal migration, and eventually, symptoms associated with reduced muscle function (15-17). In the present study, we found that the plasma levels of UNC45A were significantly lower in samples from patients with POP than those in healthy controls. Since weaken or decline of muscle cell may be the cause of POP, the role of UNC45A in the pathogenesis of POP should be explored in further research.

Researchers have investigated a number of potential diagnostic biomarkers for POP over recent years. A metabolomics screening study, involving samples of serum and urine, found that pathways involving arginine biosynthesis and purine metabolism were significantly related to POP. A total of six metabolites, including GPC, 1-methyladenosine, maleic acid, L-pyroglutamic acid, inosine, and citrate, showed significant differences when compared between patients with POP and healthy controls. These six metabolites were therefore considered as potential biomarkers for the diagnosis of POP. However, the sample size in this previous study was insufficient, with a total of 50 samples (including 24 samples in the POP group and 25 samples in the control group). Further studies are needed to fully evaluate the diagnostic efficiency of these metabolites (7). Matrix metalloproteinase (MMPs) is a protease that is related to the degradation of collagen and elastin. Studies have shown that compared with a control group, the collagen content in samples of vaginal tissue from women experiencing were decreased and that the expression levels of MMP-2 and MMP-9 were increased. These results indicated that MMPs participate in the degradation of collagen in pelvic supporting structures, thus leading to the weakening of connective tissues, and ultimately to POP (18). However, the biomechanical changes related to POP may also be the cause of the increased expression levels of MMPs. Therefore, the potential for MMP-1, MMP-2, and MMP-9, to act as biomarkers for POP needs to be confirmed in future research (19).

Data in this study provides a research basis to further develop rapid detection technology for early screening and community health care. We will start a larger size study to identify high-risk individuals and test plasma levels of HSP10, ZC3H8, and UNC45A in the followup population, to provide scientific evidence for early diagnosis. The timely and accurate biochemical detection at an early stage is very important if we are to prevent and control POP in a more effective manner. China has the largest population of any country in the world. It is estimated that there will be a huge explosion in the elderly population by 2050 , with approximately 400 million people (26.9\% of the total population) over 65 years-of-age, and approximately 150 million over 80 years-of-age. As life expectancy continues to grow, China will inevitably become one of the countries with the highest proportion of elderly individuals. Consequently, the need to prevent, control, and treat POP will become increasingly more prominent in the future since the incidence of POP will inevitably increase, potentially far higher than the incidence of other chronic diseases (20).

\section{Conclusions}

In the present study, we aimed to identify specific biomarkers for the diagnosis of POP by identifying significant differences in the expression levels of key proteins between patients with POP and healthy controls. By applying a screening assay and a quantifying the plasma levels of selected proteins, we demonstrated that HSP10, ZC3H8, and UNC45A, represent potential biomarkers for POP with good levels of accuracy. The diagnosis of POP remains a clinical challenge given that the current diagnosis of POP depends on the observation of clinical symptoms, functional testing of the pelvic floor muscles and image detection including computer tomography (CT), magnetic resonance imaging (MRI), and ultrasound scanning. These methods provide an indication of POP but are more effective in the latter phases of POP since the pathogenesis 
of POP is a chronic cumulative process involving many factors. Developing simple, fast, and effective, detection technologies for early screening and community health care will help us to identify high-risk individuals, and provide scientific evidence for early diagnosis, intervention, and treatment.

\section{Acknowledgments}

Funding: This work was supported by grants from Key Research and Development Projects of Sichuan Science and Technology Department (2021YFS0015) and Key Research and Development Plan of Ministry of Science and Technology (2021YFC2009100).

\section{Footnote}

Reporting Checklist: The authors have completed the STARD reporting checklist. Available at https://dx.doi. org/10.21037/atm-21-1607

Data Sharing Statement: Available at https://dx.doi. org/10.21037/atm-21-1607

Peer Review File: Available at https://dx.doi.org/10.21037/ atm-21-1607

Conflicts of Interest: All authors have completed the ICMJE uniform disclosure form (available at https://dx.doi. org/10.21037/atm-21-1607). The authors have no conflicts of interest to declare.

Ethical Statement: The authors are accountable for all aspects of the work in ensuring that questions related to the accuracy or integrity of any part of the work are appropriately investigated and resolved. The study was conducted in accordance with the Declaration of Helsinki (as revised in 2013). The study was approved by the Research Medical Ethics Committee of West China Second University Hospital, Sichuan University (2020121) and informed consent was taken from all individual participants.

Open Access Statement: This is an Open Access article distributed in accordance with the Creative Commons Attribution-NonCommercial-NoDerivs 4.0 International License (CC BY-NC-ND 4.0), which permits the noncommercial replication and distribution of the article with the strict proviso that no changes or edits are made and the original work is properly cited (including links to both the formal publication through the relevant DOI and the license). See: https://creativecommons.org/licenses/by-nc-nd/4.0/.

\section{References}

1. Allen-Brady K, Norton PA, Hill AJ, et al. Risk of pelvic organ prolapse treatment based on extended family history. Am J Obstet Gynecol 2020;223:105.e1-8.

2. Mattsson NK, Karjalainen PK, Tolppanen AM, et al. Pelvic organ prolapse surgery and quality of life-a nationwide cohort study. Am J Obstet Gynecol 2020;222:588.e1-10.

3. Weintraub AY, Glinter H, Marcus-Braun N. Narrative review of the epidemiology, diagnosis and pathophysiology of pelvic organ prolapse. Int Braz J Urol 2020;46:5-14.

4. Lee UJ, Kerkhof MH, van Leijsen SA, et al. Obesity and pelvic organ prolapse. Curr Opin Urol 2017;27:428-34.

5. Campeau L, Gorbachinsky I, Badlani GH, et al. Pelvic floor disorders: linking genetic risk factors to biochemical changes. BJU Int 2011;108:1240-7.

6. Vergeldt TF, Weemhoff $M$, IntHout J, et al. Risk factors for pelvic organ prolapse and its recurrence: a systematic review. Int Urogynecol J 2015;26:1559-73.

7. Deng W, Rao J, Chen X, et al. Metabolomics study of serum and urine samples reveals metabolic pathways and biomarkers associated with pelvic organ prolapse. J Chromatogr B Analyt Technol Biomed Life Sci 2020;1136:121882.

8. Zou W, Wen X, Xie C, et al. LC-Q-TOF-MS based plasma metabolomic profile of subclinical pelvic inflammatory disease: a pilot study. Clin Chim Acta 2018;483:164-9.

9. Madhu C, Swift S, Moloney-Geany S, et al. How to use the Pelvic Organ Prolapse Quantification (POP-Q) system? Neurourol Urodyn 2018;37:S39-43.

10. Bump RC, Mattiasson A, Bø K, et al. The standardization of terminology of female pelvic organ prolapse and pelvic floor dysfunction. Am J Obstet Gynecol 1996;175:10-7.

11. Kayani AC, Close GL, Dillmann WH, et al. Overexpression of HSP10 in skeletal muscle of transgenic mice prevents the age-related fall in maximum tetanic force generation and muscle Cross-Sectional Area. Am J Physiol Regul Integr Comp Physiol 2010;299:R268-76.

12. Shan YX, Liu TJ, Su HF, et al. Hsp10 and Hsp60 modulate Bcl-2 family and mitochondria apoptosis signaling induced by doxorubicin in cardiac muscle cells. J Mol Cell Cardiol 2003;35:1135-43.

13. Zou Q, Gang K, Yang Q, et al. The CCCH-type zinc 
finger transcription factor $\mathrm{Zc} 3 \mathrm{~h} 8$ represses NF- $\mathrm{NB}$ mediated inflammation in digestive organs in zebrafish. $\mathrm{J}$ Biol Chem 2018;293:11971-83.

14. Ni W, Odunuga OO. UCS proteins: chaperones for myosin and co-chaperones for Hsp90. Subcell Biochem 2015;78:133-52.

15. Epping MT, Meijer LA, Bos JL, et al. UNC45A confers resistance to histone deacetylase inhibitors and retinoic acid. Mol Cancer Res 2009;7:1861-70.

16. Liu L, Srikakulam R, Winkelmann DA. Unc45 activates Hsp90-dependent folding of the myosin motor domain. J Biol Chem 2008;283:13185-93.

17. Lehtimäki JI, Fenix AM, Kotila TM, et al. UNC-45a

Cite this article as: Wang T, Liu Y, Mei L, Cui T, Wei D, Chen Y, Zhang X, Gao L, Zhang S, Guo L, Yang P, Niu X. Proteins in plasma as a potential biomarkers diagnostic for pelvic organ prolapse. Ann Transl Med 2021;9(14):1117. doi: 10.21037/atm-21-1607 promotes myosin folding and stress fiber assembly. J Cell Biol 2017;216:4053-72.

18. Wu JM, Visco AG, Grass EA, et al. Matrix metalloproteinase- 9 genetic polymorphisms and the risk for advanced pelvic organ prolapse. Obstet Gynecol 2012;120:587-93.

19. Dviri $M$, Leron E, Dreiher J, et al. Increased matrix metalloproteinases-1,-9 in the uterosacral ligaments and vaginal tissue from women with pelvic organ prolapse. Eur J Obstet Gynecol Reprod Biol 2011;156:113-7.

20. Iglesia CB, Smithling KR. Pelvic organ prolapse. Am Fam Physician 2017;96:179-85. 